' Division of Endocrinology, University of Buenos Aires, Buenos Aires, Argentina ${ }^{2}$ Department of Pathology, University of Buenos Aires, Buenos Aires, Argentina

\author{
Correspondence to: \\ Fabián Pitoia \\ Division of Endocrinology, \\ University of Buenos Aires, \\ Córdoba 2351, C 1120 \\ Buenos Aires, Argentina \\ fpitoia@intramed.net \\ Received on Sept/29/2020 \\ Accepted on Dec/2/2020

\section{Dramatic clinical response to dabrafenib plus trametinib in anaplastic thyroid carcinoma and the challenges faced during the COVID-19 pandemic}

\author{
Fernanda Bueno' ${ }^{1}$ \\ https://orcid.org/0000-0001-9333-4576 \\ Erika Abelleira' \\ https://orcid.org/0000-0001-5217-3294 \\ Florencia von Stecher ${ }^{2}$ \\ https://orcid.org/0000-0002-9629-8459 \\ Andrea Paes de Lima ${ }^{2}$ \\ https://orcid.org/0000-0001-7190-6182 \\ Fabián Pitoia ${ }^{1}$ \\ https://orcid.org/0000-0002-2742-7085
}

\begin{abstract}
SUMMARY
Anaplastic thyroid carcinoma is the rarest tumor of the thyroid gland, representing less than $2 \%$ of clinically recognized thyroid cancers. Typically, it has an extremely rapid onset, fatal outcomes in most cases, and a median overall survival of 3 to 10 months despite aggressive multidisciplinary management. The presence of targetable mutations in anaplastic thyroid carcinoma patients is an opportunity for treatment when conventional therapeutics approaches are not effective, a frequent situation in the majority of patients. We present our experience in the management of a patient with unresectable anaplastic thyroid cancer who had a remarkable and rapid response to treatment with dabrafenib and trametinib during the COVID-19 pandemic. After four weeks of dabrafenib $150 \mathrm{mg}$ twice daily plus trametinib $2 \mathrm{mg}$ daily, he showed a dramatic reduction of the cervical mass around $90 \%$. Nearly eight weeks under treatment with dabrafenib plus trametinib, the patient remains with minimal locoregional disease without distant metastases. Arch Endocrinol Metab. 2021;65(2):242-7
\end{abstract}

\section{INTRODUCTION}

A naplastic thyroid carcinoma (ATC) is the rarest tumor of the thyroid gland, representing less than $2 \%$ of clinically recognized thyroid cancers $(1,2)$. Typically, it has an extremely rapid onset and fatal outcomes in most cases, accounting for more than half of all deaths attributable to thyroid tumors (3). At the time of diagnosis, most cases are associated with extensive local disease, and the presence of distant metastasis is observed in $20 \%$ to $50 \%$ of patients (4). The most common metastatic sites are the lungs, followed by intrathoracic and neck lymph nodes (5). Until 2018, ATC was associated with a median overall survival (OS) of 3 to 10 months, a median 1 -year survival of $20 \%$, and a median 10-year OS of less than $2 \%$, despite aggressive multidisciplinary management (6-11). The most recent database analysis reported ATC was associated with an improvement in median OS in the last three years (12).

ATC's locoregional invasiveness may cause compressive symptoms, such as dysphagia, dyspnea, stridor, and pain, which generally make ATC unresectable. Therefore, the diagnostic and therapeutic management of this entity represents a challenging situation, and a multidisciplinary team with experience in ATC management is always required (13).

According to the American Joint Committee on Cancer, ATC is classified as Stage IV, regardless of tumor size or the presence of lymph node or distant metastasis: Stage IVA describes all tumors confined to the gland; Stage IVB represents ATC with gross extrathyroidal extension; and in Stage IVC, the tumor has already spread to distant sites $(14,15)$. 
The American Thyroid Association's (ATA) guidelines consider surgical resection, when feasible, as first-line treatment and external beam radiation therapy for local control (16). ATC is a rare and aggressive tumor, thus predicting patient clinical therapy responsiveness is still challenging. Several genetic mutations have been described in ATC, that are involved in different molecular pathways linked to tumor progression, and novel therapies acting on these molecular pathways have been investigated to improve the quality of life in these patients $(15,17,18)$. Mutation of the tumor suppressor p53 gene is commonly detected among ATC (70-88\%), whereas the gene is found less frequently in follicular thyroid cancer and papillary thyroid cancer (PTC) (19). Alternatively, approximately $20-50 \%$ of anaplastic thyroid tumors harbor the BRAF (V600E) mutation (20-23).

To assess the efficacy and safety of $B R A F$ plus $M E K$ inhibition, an open-label Phase II trial evaluated dabrafenib plus trametinib in patients with nine rare tumor types, including patients with ATC. The objective response rate (ORR) was 67\%, including two complete responses. In the subset of patients with centrally confirmed $B R A F$ (V600E) mutation-positive tumors, the ORR was $75 \%$. The median progressionfree survival (PFS) was 13.8 months, and the median OS was 19.8 months (24). This multicenter phase II trial led to approval by the United States Food and Drug Administration in May 2018 for this combination of drugs for patients with BRAF (V600E)-mutated ATC with locally advanced, unresectable, or metastatic ATC with no locoregional treatment options.

The presence of targetable mutations in ATC patients is an opportunity for treatment when conventional therapeutics approaches are not effective, a frequent situation in the majority of patients (25). However, the management of these patients represents a challenge in Latin America, considering the underlying resource limitations of the health system, whether for the access to genomic testing or the subsequent targeted therapy. We previously reported how the advances in this scenario changed the outcomes. In our cohort of advanced thyroid cancer with targetable mutations, only $32 \%$ of patients who were offered the genomic test could afford to pay for the test or have the test covered by their health insurance $(26,27)$. Considering the three patients with unresectable BRAF (V600E) positive ATC, two patients received dabrafenib plus trametinib (D$\mathrm{T})$ with significant clinical benefits, but one patient died due to rapidly progressive disease before health insurance authorized D-T (27).
In this paper, we present our experience in the management of a patient with unresectable ATC with a remarkable and rapid response to treatment with $\mathrm{D}-\mathrm{T}$ during the COVID-19 pandemic.

\section{CLINICAL CASE}

A 62-year-old man with a 5-year history of a locally advanced persistent PTC was referred to our hospital in March 2020 with a rapidly growing cervical mass. $\mathrm{He}$ underwent total thyroidectomy and remnant ablation in 2015. The same year, he received an additional therapeutic radioiodine dose of $250 \mathrm{mCi}$. In June 2018, he evolved with a structural incomplete response in the left lateral neck region, with fine-needle aspiration confirming a metastatic papillary thyroid carcinoma. The patient discontinued the follow-up for one year. Four years after the initial diagnosis (July 2019), he underwent a new surgery for a 45 x $35 \mathrm{x}$ $20 \mathrm{~mm}$ cervical lesion (VI level), with incomplete resection due to tracheal adhesion. In August 2019, he received the third therapeutic dose of radioactive iodine $(150 \mathrm{mCi})$, with a post-dose whole-body scan showing only a faint uptake in the right lateral neck. Subsequently, the patient developed a rapidly growing cervical mass 2 months before he was referred to our hospital.

On clinical examination, a mass was palpated at the anterior cervical region, with increased consistency, a smooth surface, and adherence to the deep planes, including generalized inflammation (lymphangitis) around the mass and extending to the anterior thoracic region (Figures $1 \mathrm{~A}$ and B). Vital signs were normal. He mentioned dysphagia to solid food. He denied fever, dyspnea, or other symptoms of local compression.

\section{COMPLEMENTARY EXAMINATIONS}

His baseline investigations were normal, including thyroid function tests. Infection and microbiology workup all came out as negative. Computed tomography (CT) scans of the neck, chest, and abdomen were performed. The neck CT revealed an unresectable lesion of $130 \times 120 \mathrm{~mm}$ (APxT) involving the trachea, esophagus, carotid artery, jugular vein, and extending to the thoracic inlet (Figures 2A and B). This lesion measured $62 \times 42 \mathrm{~mm}$ on a CT scan performed 2 months before the patient's admission at our institution. 

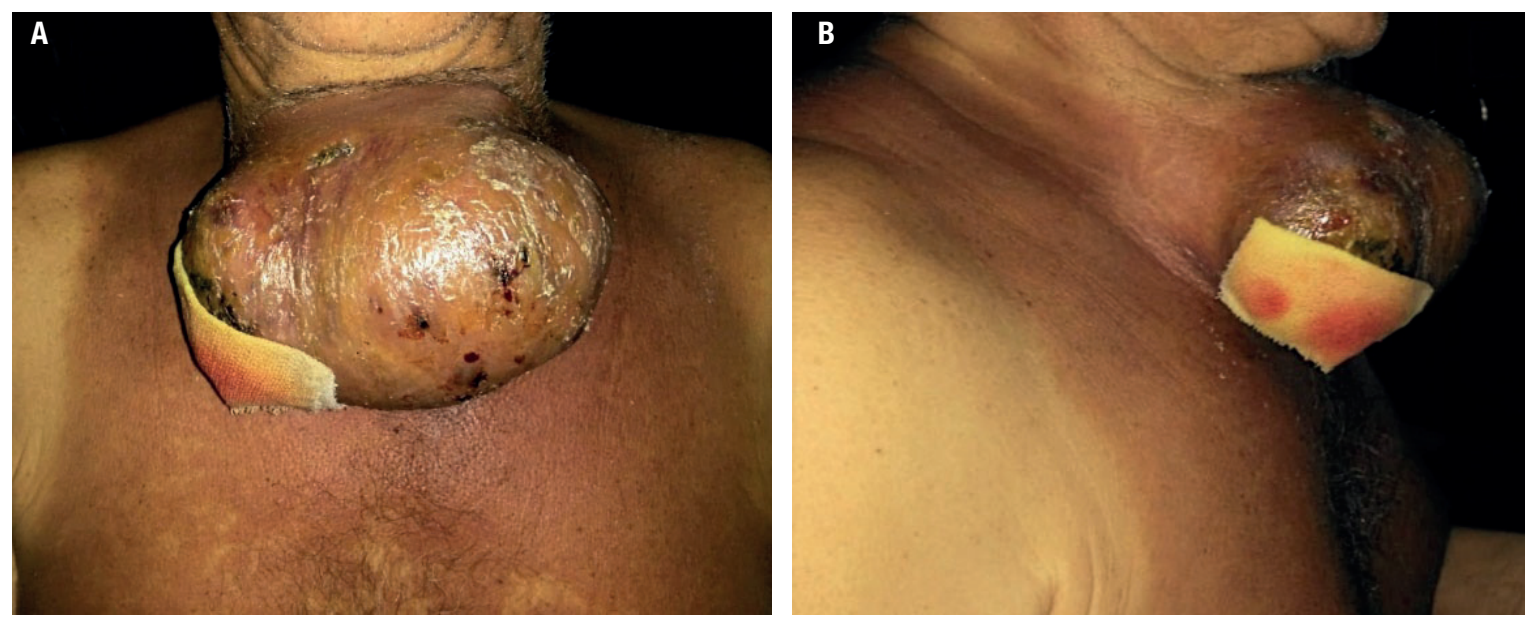

Figure 1. (A and B): Pictures of the patient's neck in the first clinical examination (large anterior cervical mass). Generalized inflammation (lymphangitis) was noted around the mass and extending to the anterior thoracic region.



Figure 2. (A and B): Axial view of Computed Tomography (CT) scans of the neck showing the cervical mass before treatment: $120 \times 130 \mathrm{~mm}$ in the thyroid and left lateral neck. (C and $\mathbf{D})$ : Four weeks after treatment, the axial view of CT scans showed a dramatic reduction of the cervical mass around $90 \%$.

The cervical lesion was biopsied by fine-needle aspiration and revealed neoplastic cells, with marked nuclear pleomorphism, macrokaryosis, evident nucleoli multinucleation, isolated bizarre nuclei, and moderate cytoplasm in a background of lymphocytes and neutrophils. However, the material was not suitable for the molecular study. Therefore, an excisional biopsy was performed, which showed an undifferentiated malignancy with pleomorphic cells in a diffuse pattern, bizarre nuclei, and multinucleation. Mitosis was frequent, and extensive areas of necrosis, hemorrhage and suppuration were observed (Figure 3 ). The biopsy finally confirmed the presence of ATC. ATC

He was tested for the BRAF status through nextgeneration sequencing on a tumor tissue sample, confirming the presence of a BRAF (V600E) mutation.

\section{Outcome and follow-up}

The tumor was considered unresectable due to tracheal involvement and extensive disease surrounding the carotid; he was not a candidate for primary surgical resection. 


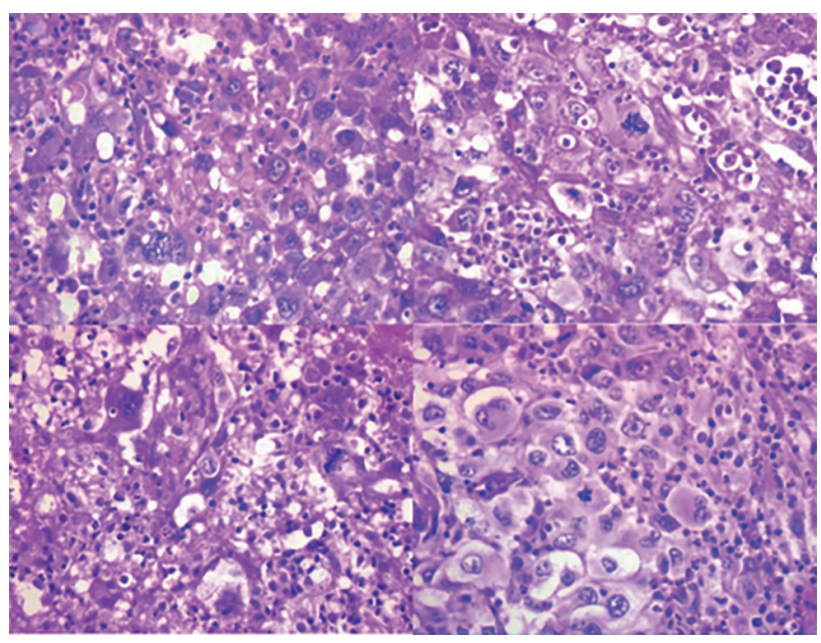

Figure 3. ATC. Pleomorphic tumor cells with atypical mitosis admixed with neutrophils (hematoxylin-eosin, X400).

As D-T was not immediately available, off-label lenvatinib $10 \mathrm{mg} /$ day was used as bridging therapy. Ten days later, the development of bleeding, minimal ulceration in the lesion, and fever made it necessary to withdraw lenvatinib treatment. In the context of the COVID-19 pandemic, the patient was swabbed to rule out a SARS-CoV-2 infection.

On June 2, the patient began the combination treatment: dabrafenib $150 \mathrm{mg}$ twice daily plus trametinib 2 mg daily. At 48 hours, the patient evolved with improvement in cervical pain and discontinued opioid intake. At 72 hours, there was evidence of tumor lesion necrosis. After only one week of therapy, the patient had a marked reduction of approximately $50 \%$, with a total resolution of dysphagia. The cervical lesion evolved with extensive necrosis and tracheal exposure; the patient was reevaluated for oncological surgery and otorhinolaryngology service, which indicated flat cures (Figure 4).

During the treatment with D-T, the patient presented adverse effects: (i) hyperglycemia (blood glucose level of $235 \mathrm{mg} / \mathrm{dl}$, before treatment blood glucose levels ranged from 110 to $124 \mathrm{mg} / \mathrm{dL}$ ), for which reason he was evaluated by the Diabetes Care Unit, they indicated dietary treatment and started treatment with metformin; (ii) fatigue grade 2 ; and (iii) constipation. The patient had a performance status grade 2 .

Additionally, the patient presented fever associated with odynophagia, which led us to rule out SARSCoV-2 several times during the treatment with D-T. Finally, the fever resolved with physical methods and antipyretic drugs, and we considered the fever as an adverse effect of D-T treatment.
Four weeks after treatment, an axial view of neck CT scans showed a dramatic reduction of the cervical mass of approximately 90\% (Figures 2C and D). The patient remains with a minimal locoregional disease without distant metastases, nearly eight weeks under treatment with D-T.

\section{DISCUSSION}

The aggressive behavior of ATC confers a rapidly fatal outcome. The immediate cause of death in most patients is related to local complications, such as airway obstruction, catastrophic hemorrhage, or circulatory failure due to compression of mediastinal vasculature $(9,10)$. Locally advanced disease also leads to severe pain and dysphagia. In a limited subset of patients with resectable disease at presentation, surgical resection has been associated with improved survival. However, for most patients who present with advanced $\mathrm{T} 4 \mathrm{~b}$ disease, radical resection is associated with increased morbidity and is not advocated because of rapid recurrence, distant disease, and poor survival outcomes.

$B R A F$ (V600E) point-activating mutation occurs in approximately $20-50 \%$ of ATC (20-23). Additionally, other targetable mutations have been found in ATC: RET/PTC rearrangements and N-TRK rearrangements, which led to therapeutical success in isolated case reports $(28,29)$.

Fortunately, the therapeutic management of ATC has expanded with the appearance of the first combination of selective $B R A F$ plus $M E K$ inhibitor therapy approved for mutated ATC BRAF (V600E), with high response rates and a significant improvement in survival (24). Some patients with initially unresectable disease may become resectable and could be considered for surgery if they respond to anti $B R A F / M E K$ combination therapy. Wang and cols. reported the first series in the literature of BRAF (V600E)-mutated ATC patients with locoregionally advanced disease treated with DT followed by surgical resection. They demonstrated the feasibility for complete tumoral resection, decreased need for tracheostomy, high pathologic response rates, and durable locoregional control (30). We reported a patient with locally advanced and metastatic anaplastic thyroid carcinoma with a BRAF (V600E) mutation with complete surgical resection after D-T treatment (26).

A recently published retrospective cohort study included 479 patients with ATC from January 2000 to October 2019, which were divided into three groups 

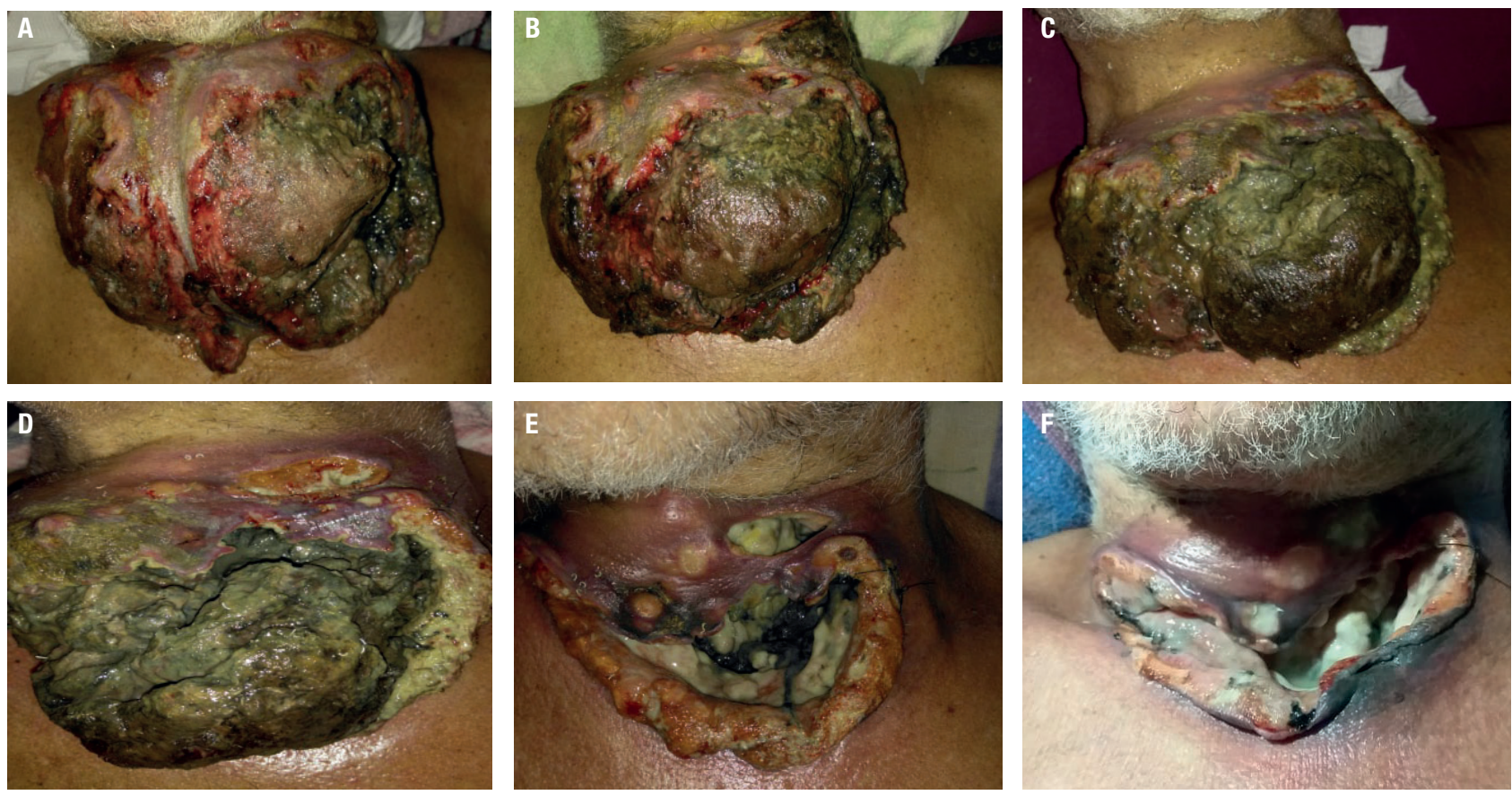

Figure 4. Lesion outcome under treatment with D-T. A: before treatment. B: one day under treatment. C: four days under treatment. D: seven days under treatment. E: ten days under treatment. F: four weeks under treatment.

according to the date of presentation: 2000-2013, 2014-2016, and 2017-2019. The median OS of the entire cohort was 9.5 months. The OS at 2 years was $18 \%$ in the $2000-2013$ group, and $42 \%$ in the 2017-2019 group, respectively $(P<.001)$. Patients undergoing surgery following neoadjuvant $B R A F$ directed therapy had a 94\% 1-year survival, with a median follow-up of 1.21 years. They concluded that the changes in patient management, such as molecularbased personalized therapies, appear to be associated with a significant increase in survival (12).

A multidisciplinary team with experience in ATC must carry out the diagnostic and therapeutic management of this entity. This situation represents a challenge in Latin American considering the underlying resource limitations. On the other hand, the management of this patient was more difficult in the context of the COVID-19 pandemic. Thus, in the presence of fever on two occasions, the viral infection had to be ruled out.

This is a report that contributes to illustrate the feasibility and effectiveness of a neoadjuvant approach using $\mathrm{D}$-T in one patient with locoregionally advanced BRAF (V600E)-mutated ATC. Further studies are required to evaluate systematically the effect of this strategy on overall survival, progression-free survival, and quality of life.
Ethical approval: the Institutional Review Board approved this study.

Informed consent: informed consent was obtained from the patient included in the case report.

Disclosure: no potential conflict of interest relevant to this article was reported.

\section{REFERENCES}

1. Neff RL, Farrar WB, Kloos RT, Kenneth BD. Anaplastic thyroid carcinoma. Endocrinol Metab Clin North Am. 2008;37(2):525-38.

2. Green LD, Mack L, Pasieka JL. Anaplastic thyroid cancer and primary thyroid lymphoma: a review of these rare thyroid malignancies. J Surg Oncol. 2006;94(8):725-36.

3. Ragazzi M, Ciarrocchi A, Sancisi V, Gandolfi G, Bisagni A, Piana S. Update on anaplastic thyroid carcinoma: morphological, molecular, and genetic features of the most aggressive thyroid cancer. Int J Endocrinol. 2014;2014:790834.

4. Giuffrida D, Gharib H. Anaplastic thyroid carcinoma: current diagnosis and treatment: Ann Oncol. 2000;11(9):1083-9.

5. Besic N, Gazic B. Sites of metastases of anaplastic thyroid carcinoma: autopsy findings in 45 cases from a single institution. Thyroid. 2013;23(6):709-13.

6. Smallridge RC, Marlow LA, Copland JA. Anaplastic thyroid carcinoma: molecular pathogenesis and emerging therapies. Endocr Relat Cancer. 2009;16(1):17-44.

7. Smallridge RC, Copland JA. Anaplastic thyroid carcinoma: pathogenesis and emerging therapies. Clin Oncol (R Coll Radiol). 2010;22(6):486-97.

8. Brignardello E, Palestini N, Felicetti F, Castiglione A, Piovesan $A$, Gallo $M$, et al. Early surgery and survival of patients with 
anaplastic thyroid carcinoma: analysis of a case series referred to a single institution between 1999 and 2012. Thyroid. 2014;24(11): 1600-6.

9. Prasongsook N, Kumar A, Chintakuntlawar AV, Foote RL, Kasperbauer J, Molina J, et al. Survival in Response to Multimodal Therapy in Anaplastic Thyroid Cancer. J Clin Endocrinol Metab. 2017;102(12):4506-14.

10. Wendler J, Kroiss M, Gast K, KreissI MC, Allelein S, Lichtenauer U, et al. Clinical presentation, treatment and outcome of anaplastic thyroid carcinoma: results of a multicenter study in Germany. Eur J Endocrinol. 2016;175(6):521-9.

11. Are C, Shaha AR. Anaplastic thyroid carcinoma: biology, pathogenesis, prognostic factors, and treatment approaches. Ann Surg Oncol. 2006;13(4):453-64.

12. Maniakas A, Dadu R, Busaidy NL, Wang JR, Ferrarotto R, Lu C, et al. Evaluation of Overall Survival in Patients With Anaplastic Thyroid Carcinoma, 2000-2019. JAMA Oncol. 2020;6(9):1-8.

13. Molinaro E, Romei C, Biagini A, Sabini E, Agate L, Mazzeo S, et al. Anaplastic thyroid carcinoma: From clinicopathology to genetics and advanced therapies. Nat Rev Endocrinol. 2017; 13(11):644-60.

14. O' Neill JP, Shaha AR. Anaplastic thyroid cancer. Oral Oncol. 2013;49(7):702-6.

15. Smallridge RC, Ain KB, Asa SL, Bible KC, Brierley JD, Burman KD, et al.; American Thyroid Association Anaplastic Thyroid Cancer Guidelines Taskforce. American Thyroid Association guidelines for management of patients with anaplastic thyroid carcinoma. Thyroid. 2012;22(11):1104-39.

16. Glaser SM, Mandish SF, Gill BS, Balasubramani GK, Clump DA, Beriwal S. Anaplastic thyroid cancer: Prognosis factors, patterns of care, and overall survival. Head Neck. 2016;38:E2083-90. https:// doi.org/10.1002/hed.24384.

17. Antonelli A, Fallahi P, Ferrari SM, Ruffilli I, Santini F, Minuto M, et al. New targeted therapies for thyroid cancer. Curr Genomics. $2011 ; 12(8): 626-31$.

18. Antonelli A, Ferrari SM, Elia G, Patrizio A, Fallahi P. Metastases free thyroid cancer patients harbouring TERT mutations may benefit from a more intensive treatment and follow-up. Gland Surg. 2019;8(3):298-300.

19. Quiros RM, Ding HG, Gattuso P, Prinz RA, Xu X. Evidence that one subset of anaplastic thyroid carcinomas are derived from papillary carcinomas due to BRAF and p53 mutations. Cancer. 2005;103(11):2261-8.
20. Kunstman JW, Juhlin CC, Goh G, Brown TC, Stenman A, Healy $J M$, et al. Characterization of the mutational landscape of anaplastic thyroid cancer via whole-exome sequencing. Hum Mol Genet. 2015;24(8):2318-29.

21. Landa I, Ibrahimpasic T, Boucai L, Sinha R, Knauf JA, Shah RH, et al. Genomic and transcriptomic hallmarks of poorly dierentiated and anaplastic thyroid cancers. J Clin Invest. 2016;126(3):1052-66.

22. Sandulache VC, Williams MD, Lai SY, Lu C, William WN, Busaidy $\mathrm{NL}$, et al. Real-Time Genomic Characterization Utilizing Circulating Cell-Free DNA in Patients with Anaplastic Thyroid Carcinoma. Thyroid. 2017;27(1):81-7.

23. Guerra A, Di Crescenzo V, Garzi A, Cinelli M, Carlomagno C, Tonacchera $\mathrm{M}$, et al. Genetic mutations in the treatment of anaplastic thyroid cancer: a systematic review. BMC Surg 2013; 13 Suppl 2 (Suppl 2):S44.

24. Subbiah V, Cabanillas ME, Kreitman RJ, Wainberg ZA, Cho JY, Keam B, et al. Dabrafenib and trametinib treatment in patients with locally advanced or metastatic BRAFV600-mutant anaplastic thyroid cancer. J Clin Oncol. 2018;36(1):7-13.

25. Iñiguez-Ariza NM, Jasim S, Ryder MM, Chintakuntlawar AV, Morris JC, Hilger CR, et al. Foundation One Genomic Interrogation of Thyroid Cancers in Patients With Metastatic Disease Requiring Systemic Therapy. J Clin Endocrinol Metab. 2020;105(7):e2346-57.

26. Smulever A, Barrio Lower Daniele S, Damiano G, Pitoia F. Complete surgical resection after Dabrafenib plus Trametinib treatment in a patient with locally advanced and metastatic anaplastic thyroid carcinoma with a BRAF V600E mutation. Thyroid. 2020;30(8):1224-5.

27. Pitoia F, Smulever A, Jerkovich F. "Letter to the Editor: Foundation $\mathrm{One}^{\mathrm{TM}}$ Genomic Interrogation of Thyroid Cancers in Patients with Metastatic Disease Requiring Systemic Therapy". JCEM. 2020;105(9):e3490-e3491.

28. Wirth LJ, Sherman E, Robinson B, Solomon B, Kang H, Lorch J, et al. Efficacy of Selpercatinib in RET-Altered Thyroid Cancers. N Engl J Med. 2020;383(9):825-35.

29. Hong DS, DuBois SG, Kummar S, Farago AF, Albert CM, Rohrberg KS, et al. Larotrectinib in patients with TRK fusion-positive solid tumours: a pooled analysis of three phase $1 / 2$ clinical trials. Lancet Oncology. 2020;21(4):531-40.

30. Wang JR, Zafereo ME, Dadu R, Ferrarotto R, Busaidy NL, Lu $C$, et al. Complete surgical resection following neoadjuvant dabrafenib plus trametinib in BRAF V600E-mutated anaplastic thyroid carcinoma. Thyroid. 2019;29(8):1036-43. 